\title{
Trichuris suis induces human non-classical patrolling monocytes via the mannose receptor and PKC: implications for multiple sclerosis
}

\author{
Gijs Kooij $^{1 *}$, Rens Braster ${ }^{1}$, Jasper J. Koning ${ }^{1}$, Lisa C. Laan', Sandra J. van Vliet ${ }^{1}$, Tamara Los $^{1}$, \\ Anne Marieke Eveleens', Susanne M. A. van der Pol', Elisabeth Förster-Waldl'², Kaan Boztug ${ }^{2,3}$, Alexandre Belot ${ }^{4}$, \\ Katka Szilagyi ${ }^{5}$, Timo K. van den Berg ${ }^{5}$, Jaap D. van Buul ${ }^{6}$, Marjolein van Egmond ${ }^{1}$, Helga E. de Vries ${ }^{1}$, \\ Richard D. Cummings ${ }^{7}$, Christine D. Dijkstra ${ }^{1}$ and Irma van Die ${ }^{1}$
}

\begin{abstract}
Introduction: The inverse correlation between prevalence of auto-immune disorders like the chronic neuroinflammatory disease multiple sclerosis (MS) and the occurrence of helminth (worm) infections, suggests that the helminth-trained immune system is protective against auto-immunity. As monocytes are regarded as crucial players in the pathogenesis of auto-immune diseases, we explored the hypothesis that these innate effector cells are prime targets for helminths to exert their immunomodulatory effects.

Results: Here we show that soluble products of the porcine nematode Trichuris suis (TsSP) are potent in changing the phenotype and function of human monocytes by skewing classical monocytes into anti-inflammatory patrolling cells, which exhibit reduced trans-endothelial migration capacity in an in vitro model of the blood-brain barrier. Mechanistically, we identified the mannose receptor as the TsSP-interacting monocyte receptor and we revealed that specific downstream signalling occurs via protein kinase $C$ (PKC), and in particular PKC $\delta$.
\end{abstract}

Conclusion: This study provides comprehensive mechanistic insight into helminth-induced immunomodulation, which can be therapeutically exploited to combat various auto-immune disorders.

Keywords: Auto-immunity, Multiple sclerosis, Innate immunity, Trichuris suis, Monocytes, Mannose receptor, Protein kinase C, PKC

\section{Introduction}

Multiple sclerosis (MS) is a chronic inflammatory disease of the central nervous system in which autoreactive $\mathrm{T}$ cells and monocyte-derived macrophages cause severe brain tissue damage, leading to neurological deficits $[1,2]$. Epidemiological studies have shown that there is an inverse correlation between the prevalence of MS and the occurrence of helminth (worm) infections [3]. Intriguingly, helminth-infected MS patients show significant lower number of relapses, reduced disability scores, and lower

\footnotetext{
* Correspondence: G.kooij@vumc.nl

'Department of Molecular Cell Biology and Immunology, Neuroscience Campus Amsterdam, VU University Medical Center, P.O. Box 70571007 MB Amsterdam, The Netherlands

Full list of author information is available at the end of the article
}

magnetic resonance imaging activity compared to uninfected MS subjects [4], suggesting a strong immunomodulatory effect of these helminths. Anthelmintic treatment of such patients was associated with significant increase in disease activity, as observed by both clinical and radiological observations [4]. These data indicate that probiotic helminth administration may be a promising avenue to treat MS. Indeed, recent clinical tests in MS using the porcine nematode Trichuris suis (T. suis) show encouraging results [5], and larger clinical trials are currently in progress to fully confirm the efficacy of helminth treatment of MS. To avoid the obvious inconvenience of using living parasites, recent genome and transcriptome analysis of $T$. suis [6] have helped to pave the way to identify the parasite products with immunomodulatory capacities. In line with 
this approach, we have recently shown that soluble products of T. suis (TsSP) were able to ameliorate clinical parameters in experimental autoimmune encephalomyelitis (EAE), a well-established animal model for MS [7].

To date, the underlying protective mechanisms of helminth products are being revealed and include the induction of regulatory responses in the host [4], probably via the modulation of dendritic cells (DCs), which are key regulatory players of the adaptive immune response [8]. Our previous work showed that TsSP induce Th2 responses via DCs, whereas the induction of Th1 and Th17 responses by TsSP-primed human DCs is strongly reduced $[7,9]$. Beside affecting adaptive immunity, we have recently reported that TsSP suppresses pro-inflammatory responses in monocyte-derived macrophages [10], which are key players in MS pathogenesis, as these cells are responsible for axonal loss and neurodegeneration [11]. In turn, these findings suggest that the observed beneficial effects of TsSP in MS patients may also be explained by a direct effect on innate immunity, and this hypothesis will be addressed in the current study. Key players of the human innate immune response are monocytes, which constitute around $10 \%$ of the total leukocyte pool and are found in the blood, bone marrow and spleen. They originate in the bone marrow from hematopoietic stem cells [12] and are divided into subsets based on the surface expression of CD14 and CD16 [13]. The most prevalent monocyte subset in the blood consists of classical monocytes which display high CD14 levels and low CD16 levels $\left(\mathrm{CD} 14^{++} \mathrm{CD} 16^{-}\right)$and additionally express the chemokine receptor CCR2 [14]. Other subsets include CD16-expressing monocytes, which can be further divided in two subpopulations: $\mathrm{CD} 14^{+} \mathrm{CD} 16^{+}$ (intermediate) and $\mathrm{CD} 14^{\mathrm{dim}} \mathrm{CD} 16^{++}$(non-classical), which both express high levels of the chemokine receptor $\mathrm{CX}_{3} \mathrm{CR} 1$ [15]. Of these CD16-expressing monocytes, the non-classical cells are regarded as patrolling cells, as they adhere and migrate along the luminal surface of endothelial cells that line small blood vessels, similar to the mouse GR $1^{\text {low }}$ monocyte population [16]. Genetic deletion and/or inhibitory antibody studies have shown that besides $\mathrm{CX}_{3} \mathrm{CR} 1$ [17], the patrolling behaviour of monocytes is also dependent on the integrin Lymphocyte Function-associated Antigen 1 (LFA-1;[16]), which binds to intercellular cell adhesion molecule-1 (ICAM-1) on endothelial cells [18]. In MS, the majority of monocytes display a classical (inflammatory) phenotype based on high CD40, CD86, HLA-DR, CD64 and CCR2 expression [19] and active MS lesions are dominated by monocyte-derived macrophages that have entered the CNS by traversing the blood-brain barrier (BBB). Therefore, research is needed to identify molecules and mechanisms that can skew these inflammatory monocytes into antiinflammatory [16] and wound healing [20] patrolling cells that exhibit reduced transendothelial migration capacity, and thereby provide novel ways to combat disease progression.
As monocytes belong to the first responders to helminths as well as their secreted molecules and are regarded as crucial players in various auto-immune diseases, we hypothesized that these innate effector cells are prime targets for the helminths to exert their immunomodulatory effects. In the current study, we have investigated the effects of TsSP on human monocytes and report that TsSP potently affect the classical monocyte population by inducing a shift from classical to non-classical cells with reduced CCR2 expression and eliciting a differential pro- and anti-inflammatory cytokine response. TsSP-treated cells show a patrolling phenotype and display reduced monocyte adhesion and transendothelial migration capacities in an in vitro model of the BBB. Mechanistically, we identified the mannose receptor (MR) as the dominant TsSP-interacting monocytic receptor and revealed that protein kinase $\mathrm{C}$ (PKC), and in particular PKC $\delta$ signals downstream upon TsSP treatment. Overall, these data illustrate a potent antiinflammatory effect of TsSP on human monocytes and thereby provide further mechanistic insight into the therapeutic potential of these helminth compounds in autoimmune diseases like MS.

\section{Materials and Methods}

\section{TsSP and reagents}

Soluble products of Trichuris suis (TsSP) were prepared as described previously $[9,10]$. A limulus amebocyte lysate assay (Thermo scientific, USA) was used to determine endotoxin levels in TsSP, which appeared to be similar to background levels, thereby excluding LPS contamination. Cleavage of TsSP peptide chains (using $\alpha$-chymotrypsin (CT, Sigma, USA) and oxidation of glycan moieties (using sodium periodate (PI; $10 \mathrm{mM}$, Sigma) was performed as described previously [9]. We used blocking antibodies for the human mannose receptor (MR, CD206, BD Pharmingen, USA) or Dectin-2 (Clone 545943, R\&D Systems, USA). Recombinant human TNF- $\alpha$ was obtained from Invitrogen (Carlsbad, CA, USA). To study the involvement of PKC or Rho GTPases, we used the panPKC inhibitor Bisindolylmaleimide I (GF109203, Enzo Life Sciences, the Netherlands) as well as the Rac1-GEF inhibitor NSC23766 (Bio-Techne, Abingdon, UK) and the Rho kinase inhibitor Y27632 (Sigma-Aldrich, Steinheim, Germany).

\section{Monocyte isolation}

Peripheral blood mononuclear cells (PBMCs) were isolated using Ficoll density gradient (Lymphoprep ${ }^{\mathrm{rm}}$, Axis- Shield, Oslo, Norway) from buffy coats obtained from healthy donors (Sanquin Blood Bank, Amsterdam, the Netherlands) or patients who signed informed consent based on principles outlined in the Declaration of Helsinki. The studies were approved by the local ethics committee of Sanquin Blood Supply, Medical University of Vienna, Vienna, Austria and Comitè de Protection des Personnes Sud-EST IV, France. Patients' characteristics including details about 
PRKCD gene mutations have been described before [21, 22]. Monocyte isolation was performed by gradient centrifugation on Percoll (Amersham Biosciences, USA) according to manufacturer's protocol. To increase monocyte purity, we used anti-CD14 magnetic beads (Miltenyi Biotec, Germany) with a MACS $^{\odot}$ MultiStand and LS Column by passing $3 \mathrm{ml}$ of MACS buffer ( $2 \mathrm{mM}$ EDTA and $0.1 \%$ FCS in PBS) according to the manufacturers' protocol. Monocyte purity (based on CD68 expression) was $>90 \%$ as assessed by flow cytometry performed (FACSCalibur $^{\mathrm{TM}}$ using CELLQuest ${ }^{\mathrm{TM}}$ software (BD Biosciences) and monocytes were cultured in RPMI 1640 medium supplemented with $10 \%$ heat-inactivated fetal calf serum (FCS), $2 \mathrm{mM}$ L-glutamine, $100 \mathrm{U} / \mathrm{ml}$ penicillin and $100 \mu \mathrm{g} / \mathrm{ml}$ streptomycin (all obtained from GibcoBRL Life Technologies, Breda, the Netherlands).

\section{Flow cytometry}

Freshly isolated monocytes were cultured in the presence or absence of TsSP $(40 \mu \mathrm{g} / \mathrm{ml})$ for $16 \mathrm{~h}$ and subsequently harvested. Next, monocytes were washed twice with PBS and labelled for $1 \mathrm{~h}$ at $4{ }^{\circ} \mathrm{C}$ with primary antibody (Table 1 ) diluted in PBS containing 0.1\% BSA. Cells were washed twice in PBS and incubated for $1 \mathrm{~h}\left(4{ }^{\circ} \mathrm{C}\right)$ with fluorescent labelled secondary antibodies diluted in PBS/0,5 \% BSA (FACS buffer), washed twice and resuspended in FACS buffer prior to FACS analysis. We used either four or eight colour flow cytometry. Four colour flow cytometry (FACSCalibur, Becton Dickinson, Belgium) was used in combination with Cell Quest software (Becton Dickinson) and FlowJo software version 9.4.0 for Microsoft (Tree Star, San Carlos, CA) to analyse expression of markers. For eight colour flow cytometry, we used a Cyan ADP High Performance Research Flow Cytometer (Beckman Coulter) and results were analyzed with Summit Software v4.3. Single stained cells were used to compensate for spectral overlap. Fluorescence Minus One (FMO) stained cells were used to set boundaries between positively and

Table 1 Antibodies used for FACS analysis

\begin{tabular}{|c|c|c|}
\hline Antibody & Clone & Manufacturer \\
\hline CD14-APC & MEM-15 & Immunotools, Germany \\
\hline CD16-FITC & LNK16 & Immunotools, Germany \\
\hline CCR2-biotin & 48607 & R\&D systems, USA \\
\hline $\mathrm{CX}_{3} \mathrm{CR} 1$ & ab8021 & Abcam, the Netherlands \\
\hline LFA-1 & L7 & $\begin{array}{l}\text { Dept. of Molecular Cell Biology } \\
\text { and Immunology, Amsterdam, } \\
\text { the Netherlands }\end{array}$ \\
\hline LFA-1 (affinity) & L16 & $\begin{array}{l}\text { Dept. of Molecular Cell Biology } \\
\text { and Immunology, Amsterdam, } \\
\text { the Netherlands }\end{array}$ \\
\hline LFA-1 (avidity) & Kim127 & Kind gift from MK Robinson (UK) \\
\hline CD206 (MR) & 19.2 & BD Pharmingen, USA \\
\hline
\end{tabular}

negatively stained cells. Sytox Blue dead cell stain (Molecular Probes, the Netherlands) was used to discriminate between live and dead cells.

\section{Cytokine assays}

The production of pro- and anti-inflammatory cytokines was assessed by enzyme-linked immunosorbent assay (ELISA) in cell-free supernatant samples using the Human Inflammatory 5-Plex Panel (Invitrogen, USA). This multiplex bead assay was performed according to the user manual supplied by Invitrogen. Samples were measured by Luminex 200 TM (Bio-Rad, California, USA) and analysed using Bio-plex ManagerTM 6.0 software.

\section{DHR assay}

Reactive oxygen species (ROS) production of monocytes was measured using dihydrorhodamine (DHR), which reacts with ROS in a peroxidase-like reaction to yield fluorescent rhodamine 123 [23]. After culturing of monocytes in the presence or absence of TsSP $(40 \mu \mathrm{g} / \mathrm{ml})$ for $16 \mathrm{~h}$, cells were rinsed twice with RPMI, incubated for $30 \mathrm{~min}$ at $37^{\circ} \mathrm{C}$ with $0.5 \mu \mathrm{M}$ DHR (Sigma Aldrich, Germany) in RPMI medium. After that, cells were rinsed twice with PBS/0.1 \% BSA, transferred to FACS tubes and analysed on a FACSCalibur (see above) with excitation at $488 \mathrm{~nm}$ and the emitted fluorescence collected at $525 \mathrm{~nm}$.

\section{RNA isolation and quantitative real-time PCR}

Messenger RNA (mRNA) was isolated using a mRNA Capture kit (Roche, Switzerland), and subsequently transcribed into cDNA using the Reverse Transcription System kit (Promega, USA), as described previously [24]. Quantitative real-time PCR was performed with the SYBR Green method as previously described [24]. Oligonucleotides were designed using Primer Express 2.0 (Applied Biosystems, USA) computer software. All primer sequences are listed in Table 2 and expression levels of transcripts obtained with real-time PCR were normalized to GAPDH expression levels.

\section{Western blotting}

Freshly isolated monocytes were cultured in the presence or absence of TsSP $(40 \mu \mathrm{g} / \mathrm{ml})$ for $30 \mathrm{~min}$. Cell lysates were prepared using NP-40 lysis buffer (1\% Nonidet P-40, $10 \%$ glycerol, $100 \mathrm{mM} \mathrm{NaCl}$, $10 \mathrm{mM}$ $\mathrm{MgCl}_{2}, 50 \mathrm{mM}$ Tris, $\mathrm{pH}$ 7.4) mixed with protease and phosphatase inhibitors (Halt protease and phosphatase inhibitor cocktail, Thermo Fisher Scientific, Rockford, IL, USA). Prior to immunoblotting, lysates were boiled 10 min in Laemmli sample buffer containing $1 \% \beta$ mercaptoethanol. Equal sample volumes were subjected to $10 \%$ sodium dodecyl sulfate-PAGE and transferred to nitrocellulose membranes (Schleicher \& Schuell, Dassel, Germany). Membranes were washed with TSM/ 
Table 2 Primers used for RT-PCR

\begin{tabular}{lll}
\hline Gene & Forward $\left(5^{\prime}-3^{\prime}\right)$ & Reverse $\left(5^{\prime}-3^{\prime}\right)$ \\
\hline GAPDH & CCATGTTCGTCATGGGTGTG & GGTGCTAAGCAGTTGGTGGG \\
SOCS1 & TGAACTCGCACCTCCTACCTCT & CAACCCCTGGTTGTGCAA \\
IL-10 & CGCTGTCATCGATTCTTCCCT & AGGCATTCTTCACCTGCTCCAC \\
TGF- $\beta$ & ACTATTGCTTCAGCTCCACGGA & AGTCAATGTACAGCTGCCGCA \\
TNF-a & GCCCATGTTGTAGCAAACCCT & ATGAGGTACAGGCCCTCTGATG \\
IL-6 & AATTCGGTACATCCTCGACGG & GTTGTTTCTGCCAGTGCCT \\
CD206 (MR) & GTCTTGGGCCACAGGTGAA & AAGGCGTTGGATAGCCACA \\
\hline
\end{tabular}

0,05\% Tween and blocked in TSM/0,05 \% Tween/10 \% roti-block (Techmate, Milton Keynes, UK, \#A151.4). The following primary antibodies (in TSM/0,05\% Tween $/ 5 \%$ BSA) were used: PhosphoPKC (Cell signaling, Beverly, MA, USA, \#9371S) and GAPDH (Santa Cruz Biotechnology, Heidelberg, Germany, \#sc-32233) as a loading control. Followed by incubation with polyclonal goat anti-rabbit HRP (Dako, Heverlee, Belgium, \#p0448) and polyclonal rabbit anti-mouse HRP (Dako, Heverlee, Belgium, \#p0161). Proteins were detected using SuperSignal West Pico Chemiluminescent Substrate (Thermo Scientific, USA), in an EpiChemi II Darkroom (UPV Laboratory Products). Bands were quantified using Image $\mathrm{J}$.

\section{Live cell imaging for assessment of monocyte motility}

Real-time video recordings of monocytes were performed with an inverted phase-contrast microscope (Olympus, IX81-ZDC, Suffolk, U.K.) housed in a humidified, $5 \% \mathrm{CO}_{2}$ gassed, temperature-controlled $\left(37{ }^{\circ} \mathrm{C}\right)$ chamber. For this, $3 \times 10^{5}$ freshly isolated monocytes were applied in each well of an IBIDI slide in the presence or absence of TsSP $(40 \mu \mathrm{g} / \mathrm{ml})$, and randomly selected fields were recorded for $240 \mathrm{~min}$. Pictures were taken every 3 min with an Olympus ColorView II camera (Olympus Nederland BV). Recordings were analyzed using CELL F trackIT software (Olympus Soft Imaging Solutions, Münster, Germany).

\section{Monocyte adhesion and migration assays}

The immortalized human brain endothelial cell line hCMEC/D3, which preserves the key features of brain endothelium, was cultured as described [25]. Monocyte migration of primary human monocytes across confluent monolayers of hCMEC/D3 cells was studied with timelapse video microscopy as described previously [26]. Freshly isolated monocytes were cultured in the presence or absence of TsSP $(40 \mu \mathrm{g} / \mathrm{ml})$ for $16 \mathrm{~h}$ and subsequently washed and added $\left(7.5 \times 10^{5}\right.$ cells $\left./ \mathrm{ml}\right)$ to hCMEC/D3 cells, and the number of migrated monocytes were counted after $4 \mathrm{~h}$. For monocyte adhesion experiments, the TsSP-treated or untreated monocytes were fluorescently labelled with $0.5 \mu \mathrm{M}$ BCECF-AM (Molecular Probes) for $15 \mathrm{~min}$ at $37{ }^{\circ} \mathrm{C}$. Labelled monocytes $\left(1 \times 10^{6}\right.$ cells $\left./ \mathrm{ml}\right)$ were added to confluent monolayers of hCMEC/D3 cells and allowed to adhere for $30 \mathrm{~min}$ at $37{ }^{\circ} \mathrm{C}$ and $5 \% \mathrm{CO}_{2}$. Nonadherent cells were removed by gentle washing with prewarmed medium. Adhered cells were lysed with $0.1 \mathrm{M} \mathrm{NaOH}$, and fluorescence intensity was determined (Fluostar 32, BMG; excitation $485 \mathrm{~nm}$, emission $535 \mathrm{~nm}$ ). The number of adherent monocytes was calculated using a calibration curve.

\section{Statistical analysis}

All data were analyzed statistically by means of analysis of variance (ANOVA) and Student- $t$ test. Statistical significance was defined as $* p<0.05$, ** $p<0.01$, *** $p<0.001$.

\section{Results}

TsSP induce a shift from classical to non-classical human monocytes

To identify the pathways by which $T$. suis soluble products (TsSP) affect the innate immune system, we first investigated whether TsSP causes changes in the phenotype of human blood monocytes. As shown in Fig. 1a and c, these circulating cells can be divided into two subpopulations; a large population of inflammatory CD14 expressing cells (CD14 ${ }^{++} \mathrm{CD} 16^{-}$, classical monocytes) with high CCR2 expression, and a small population of anti-inflammatory CD16 expressing cells $\left(\mathrm{CD} 14^{\mathrm{dim}} \mathrm{CD} 16^{++}\right.$, non-classical monocytes and a $\mathrm{CD} 14^{+} \mathrm{CD} 16^{+}$intermediate population) with high $\mathrm{CX}_{3} \mathrm{CR} 1$ expression. Interestingly, TsSP treatment of monocytes results in a decreased percentage of classical $\mathrm{CD} 14^{++} \mathrm{CD} 16^{-}$monocytes and an increased percentage of $\mathrm{CD} 14{ }^{\text {dim }} \mathrm{CD} 16^{++}$non-classical monocytes (Fig. 1b). Moreover, TsSP treatment significantly reduces the proportion of CCR2-positive cells in classical monocytes and slightly but not significantly induces the proportion of $\mathrm{CX}_{3} \mathrm{CR} 1$-positive cells in non-classical monocytes (Fig. 1d, e). These results indicate that TsSP predominantly affect classical monocytes, by inducing a shift from classical to non-classical cells.

To further investigate these phenotypic alterations, we analysed the effects of TsSP on gene expression levels of 


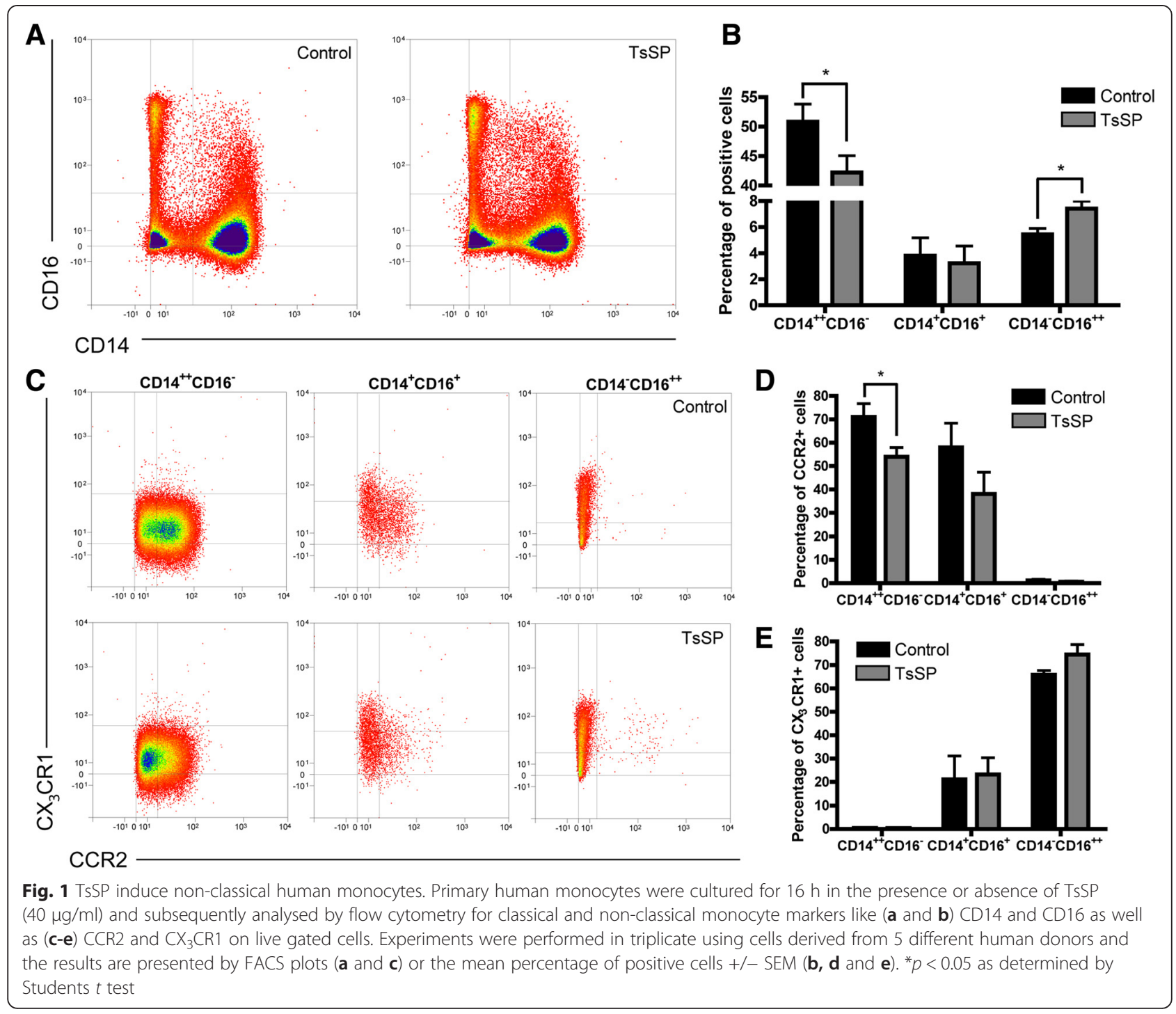

various anti-inflammatory markers including SOCS1, IL10 and TGF $\beta$, as well as pro-inflammatory cytokines like TNF- $\alpha$ and IL- 6 in purified monocytes. As shown in Fig. 2a-e, TsSP treatment significantly induces gene expression of both pro- and anti-inflammatory markers. Next, the levels of secreted IL-10, TGF $\beta$, TNF- $\alpha$ and IL6 were determined, as well as the production of reactive oxygen species (ROS), a typical marker of inflammatory monocytes [16]. In line with the transcriptional results (Fig. 2a-e), TsSP significantly enhances the secretion (Fig. 2f-i) and production (Fig. 2j) of these pro- and antiinflammatory mediators. Of note, the induction of antiinflammatory genes occurs predominantly at later time points $(16 \mathrm{~h})$ compared to the induction of proinflammatory genes $(2 \mathrm{~h})$, thereby distinguishing the acute inflammatory response to the pathogen from the apparent secondary anti-inflammatory response. Together, these results suggest that TsSP potently affect the classical monocyte population, by lowering their CCR2 expression. In turn, this may lead to a shift into non-classical cells and concomitantly elicit a differential cytokine response.

\section{TsSP induce motility behaviour in human monocytes}

It was previously shown that human non-classical monocytes have a distinct (patrolling) motility behaviour in vivo, thereby representing the human homologue of the murine patrolling $\mathrm{Gr} 1^{\text {low }}$ monocytes with anti-inflammatory capacities [16]. To test the effect of TsSP on the motility of human monocytes, we performed live cell imaging experiments. Notably, TsSP-treated monocytes display extensive crawling behaviour (Electronic supplementary material (Additional file 1: video 1 (control) and Additional file 1: video 2 (TsSP-treated cells)), showing both a higher undirected motility and more distance travelled compared to 


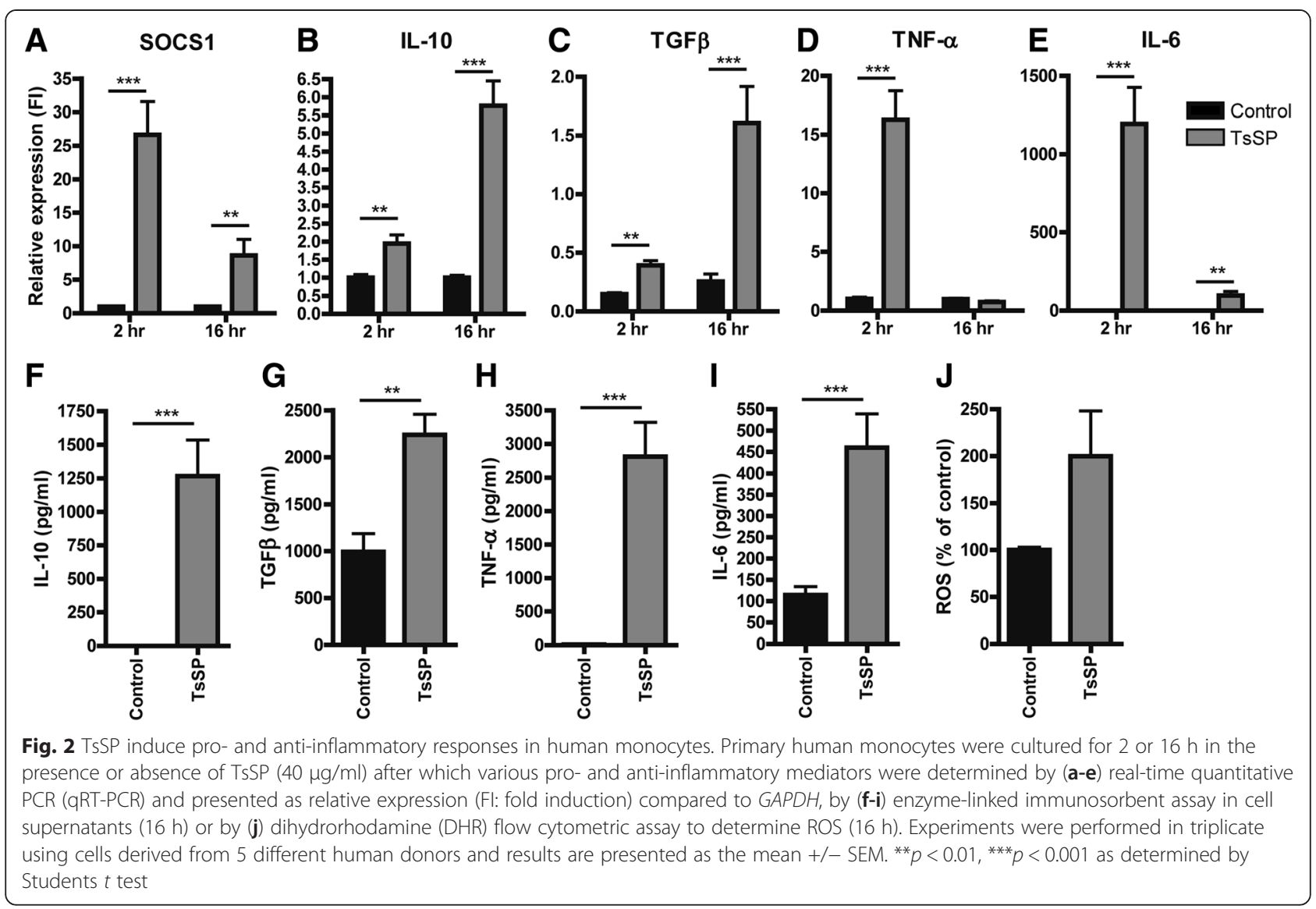

untreated cells (Fig. 3a,b). This patrolling behaviour was previously shown to be dependent on $\mathrm{CX}_{3} \mathrm{CR} 1$ [17] as well as lymphocyte function-associated antigen-1 (LFA-1, [16]). We did not observe a significant increase in $\mathrm{CX}_{3} \mathrm{CR} 1$ expression upon TsSP treatment (Fig. 1e). However, by using specific antibodies to determine LFA-1 affinity (Kim127, [27]) and avidity (L16, [28]), we found that TsSP treatment specifically increases LFA-1 avidity in human monocytes, whereas total LFA-1 as well as LFA-1 affinity levels remained unaltered (Fig. 3c). In general, cell motility is regulated by the activity of small Rho GTPases like Rho, Rac and Cdc42, which have a direct effect on the actin cytoskeleton [29]. To investigate whether the TsSPinduced monocyte motility is dependent on Rho GTPases, we performed live cell imaging experiments in the presence or absence of specific Rho GTPase inhibitors like NSC-23766 (Rac1) and Y27632 (Rho kinase inhibitor). As shown in Fig. 3d, blocking these Rho GTPases significantly limits the TsSP-induced patrolling behaviour, indicating that these GTPases play a key role in the TsSP-induced signalling pathway. Overall, these results indicate that TsSP induces a patrolling behaviour in monocytes in a Rac/Rho GTPase-dependent manner.

\section{TsSP-induced motility of human monocytes is} glycan-dependent

We have previously shown that TsSP can downregulate inflammatory responses in DCs in a glycan-dependent manner [9]. To test whether the effects we observed on monocytes after TsSP treatment are glycan- and/or protein-mediated effects, we used periodate (PI) to oxidize glycan structures on TsSP or chymotrypsin (CT) to disrupt protein structures. As shown in Fig. 4a, PI treatment of TsSP caused a profound loss of the TsSPinduced patrolling behaviour of monocytes, whereas $\mathrm{CT}$ treatment caused a small but not significant reduction. These results illustrate an important role for TsSPglycans in the induction of patrolling monocytes.

\section{TsSP-treatment of human monocytes impedes their BBB} transendothelial migration

A crucial hallmark in the pathogenesis of MS is the central nervous system (CNS) entry of leukocytes, which ultimately causes severe parenchymal tissue destruction. To enter the CNS, monocytes adhere to and finally transmigrate across brain endothelial cells (ECs) of the blood-brain barrier (BBB). To test whether TsSP 


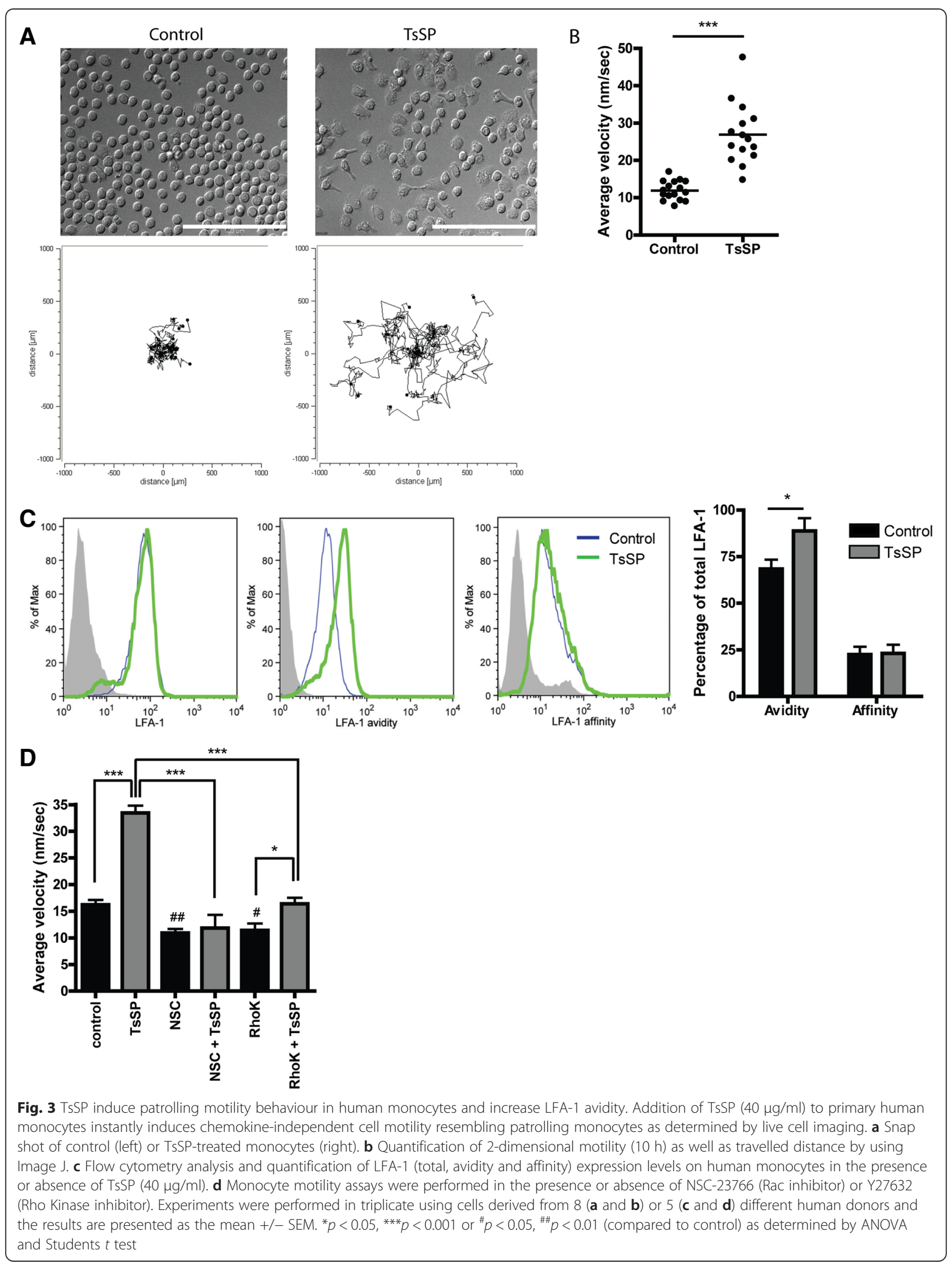



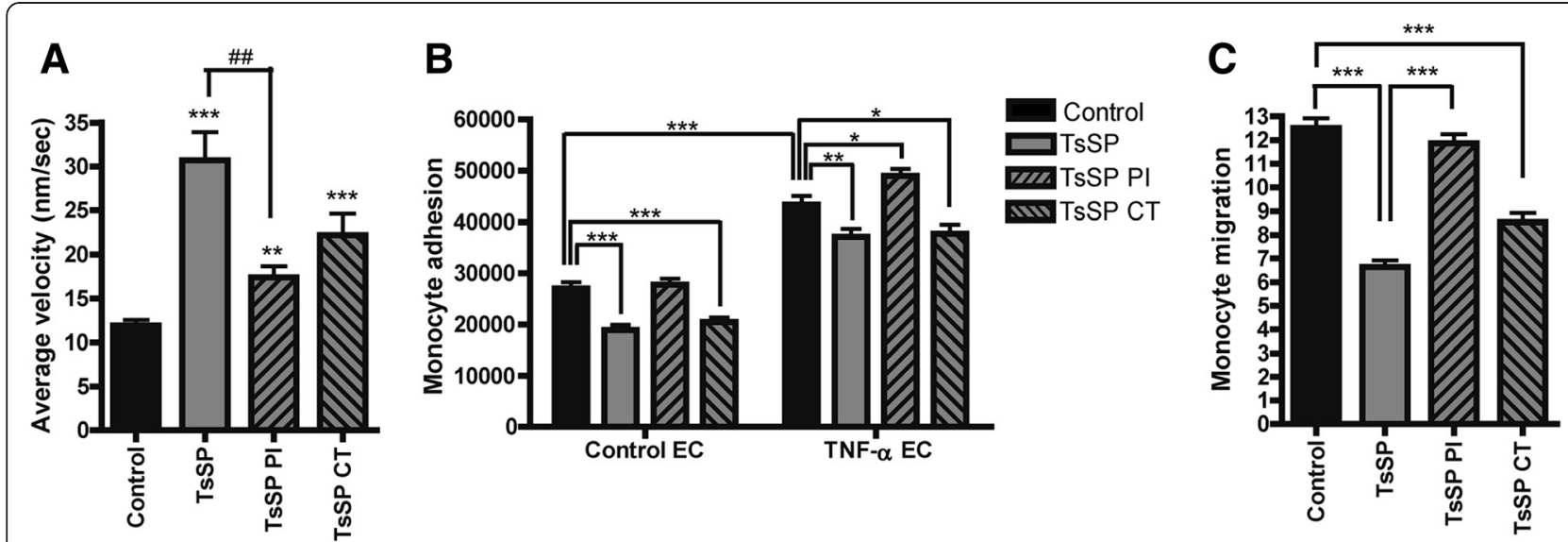

Fig. 4 TsSP-induced functional effects on monocytes are glycan-dependent. Primary human monocytes were treated with TsSP, periodate (PI)- or chymotrypsin (CT)-treated TsSP (all $40 \mu \mathrm{g} / \mathrm{ml}$ ) after which the cell motility was instantly visualized by (a) live cell imaging (10 h) and subsequently quantified by using Image J. Monocytes that were incubated with TsSP or PI/CT-TsSP for 16 h were used to study (b) monocyte adhesion to brain endothelial cells as well as (c) monocyte transendothelial migration as studied by time-lapse video microscopy. These experiments were performed on control (b) or TNF-a-treated endothelial cells (b and $\mathbf{c}$ ). Experiments were performed in triplicate using cells from 8 (a) or 5 (b and $\mathbf{c}$ ) different human donors and the results are presented as the mean $+/-$ SEM. ${ }^{*} p<0.05$, ** or ${ }^{\# \#} p<0.01,{ }^{* * *} p<0.001$ as determined by ANOVA and Students $t$ test

treatment of monocytes affects these processes, we analysed monocyte adhesion in vitro, as well as their transendothelial migration using human brain ECs [25]. To mimic the inflammatory conditions as seen in MS, we treated the ECs with the inflammatory cytokine TNF- $\alpha$, thereby inducing the expression of adhesion molecules like ICAM-1 and VCAM-1 [30], resulting in enhanced monocyte adhesion to brain EC (Fig. 4b). Importantly, TsSP treatment of monocytes significantly reduced their adhesion capacity, both under control and inflammatory conditions (Fig. 4b). In this regard, TsSP glycans also play a role, since PI treatment of TsSP completely abolished the induced reduction of monocyte adhesion (Fig. 4b). Furthermore, TsSP treatment of monocytes profoundly reduced their transendothelial migration capacity across TNF- $\alpha$-treated EC by $50 \%$ in a glycan-dependent manner (Fig. 4c). Together, these results indicate that periodate-sensitive moieties within TsSP modulate monocyte behaviour, by inducing patrolling cells and hampering their adhesion and transmigration capacity.

\section{TsSP interacts with the mannose receptor on human monocytes}

The contribution of periodate-sensitive TsSP moieties in the modulation of monocyte function suggests that glycans on TsSP may be recognized by glycan-binding receptors such as C-type lectins (CLRs), leading to an altered monocyte behaviour. We have recently shown that DCs can bind to TsSP via the mannose receptor (MR, CD206) and that TsSP are strongly bound by ConA, suggesting the presence of oligo-mannose-type glycans on TsSP [9]. Next to the MR, the TsSP glycans may also be recognized by other monocytic CLRs with mannose recognizing potential like Dectin-2 [31]. To evaluate this possibility, we first analyzed the expression of such CLRs on monocytes, which show that both the MR and Dectin-2 are expressed and that MR expression is induced upon TsSP treatment (Additional file 2). Next, we evaluated the involvement of the MR and Dectin-2 in modulating monocyte behaviour. Interestingly, treatment of monocytes with TsSP in the presence of a MR blocking antibody significantly inhibited the TsSPinduced patrolling behaviour of monocytes (Fig. 5a) and rescued the TsSP-induced reduction in monocyte transendothelial migration (Fig. 5b). In contrast, blocking of Dectin-2 did not affect monocyte patrolling behaviour and monocyte transendothelial migration (Additional file 3), suggesting that TsSP predominantly exerts its effect on human monocytes via the MR.

\section{TsSP induce PKC signaling downstream of the MR}

The MR has a short cytoplasmic tail lacking ITIM or ITAM signalling motifs [32] and its downstream signalling pathway remains largely elusive. As CCR2 expression and LFA-1 activation can be regulated by PKC [33, 34], we hypothesized that the observed effects of TsSP on CCR2 expression and LFA-1 avidity (Figs. 1c and 3c) were $\mathrm{MR}$ and PKC-dependent. Indeed, as shown in Fig. 6a, the TsSP-induced downregulation of CCR2 was significantly rescued in the presence of the panPKC inhibitor bisindolylmaleimide I (GF109203) as well as in the presence of blocking antibodies to the MR. Moreover, the TsSP-induced LFA-1 avidity was completely abolished after blocking the MR as well as in the presence of 

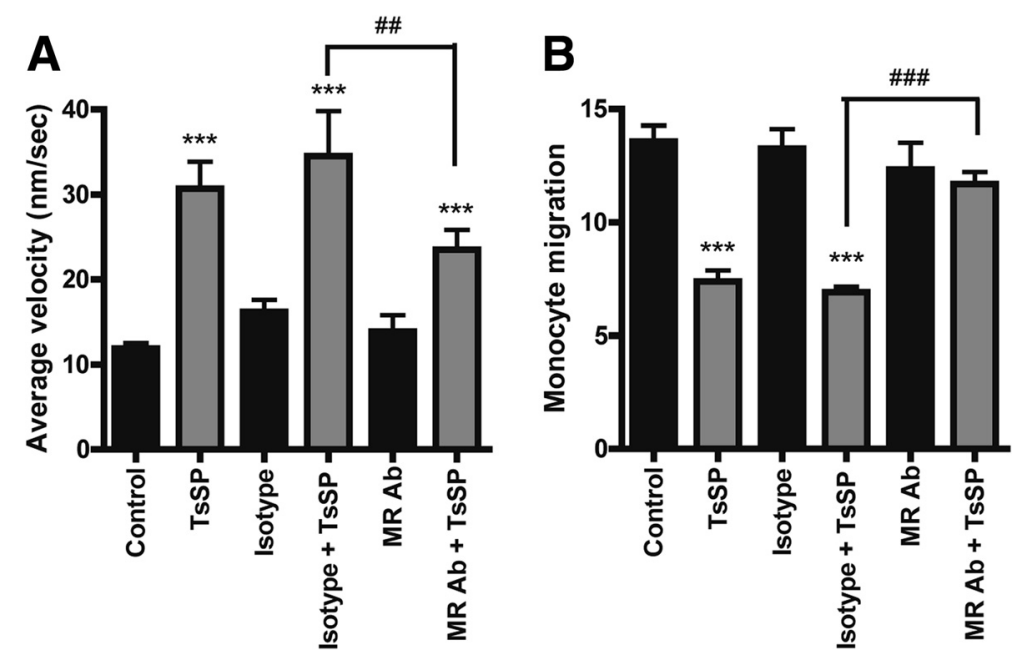

Fig. 5 TsSP interacts with the MR on human monocytes. The effect of blocking antibodies for the mannose receptor (MR, CD206) or its isotype control (both $10 \mathrm{\mu g} / \mathrm{ml}$ ) on (a) the TsSP-induced patrolling phenotype or (b) transendothelial migration capacity was tested and subsequently quantified. Experiments were performed in triplicate using cells derived from 8 (a) or 5 (b) different human donors and the results are presented as the mean $+/-$ SEM. ${ }^{* *}$ or ${ }^{\# \#} p<0.01,{ }^{* *} p<0.001$ as determined by ANOVA and Students $t$ test

GF109203 (Fig. 6b), whereas LFA-1 affinity remained unaffected (Fig. 6c). Finally, the TsSP-differential cytokine profile as evidenced by TNF- $\alpha$ and IL-10 secretion was significantly reduced after blocking the MR as well as in the presence of GF109203 (Fig. 6d, e). Together, these results indicate that TsSP interact with the MR on monocytes and affect their phenotype via PKC.

To provide evidence that TsSP directly activates PKC via the MR, we performed Western Blotting using phosphoPKC antibodies. As shown in Fig. 6f, TsSP treatment significantly induces PKC phosphorylation, to a similar level as the well-known PKC activator phorbol 12-myristate 13acetate (PMA). Importantly, this TsSP-induced PKC phosphorylation was completely abrogated in the presence of blocking antibodies to the MR, thereby providing direct evidence that TsSP induce a PKC-dependent signalling pathway via the MR in human monocytes.

\section{TsSP predominantly exerts its effect on human monocytes via PKC $\delta$}

The PKC family consists of classical (PKC $\alpha, \beta \mathrm{I}, \beta \mathrm{II}$, and $\gamma)$, novel (PKC $\delta, \varepsilon, \theta$, and $\eta)$, and atypical $(\zeta$ and $\mathrm{s} / \lambda$ ) members, and in mice it was recently suggested that PKC\& may signal downstream of the MR [35]. However, data in a human setting are lacking and research on PKC $\delta$ is complicated by the fact that specific inhibitors are currently unavailable [36]. To circumvent this and to assess the role of PKC $\delta$ in the TsSP-induced monocyte modulation, we isolated and analysed monocytes from patients with extremely rare mutations in the PRKCD gene $[21,22]$ that either lack PKC $\delta$ or have a considerably reduced expression in monocytes-derived macrophages
[36]. Importantly, the TsSP-induced patrolling behaviour in control cells was significantly impaired in $\mathrm{PKC} \delta$ deficient monocytes (Fig. 7a). PKC $\delta$-deficient monocytes showed a trend towards decreased CCR2 expression, which remained unaltered after TsSP treatment (Fig. 7b). Lack of PKC $\delta$ completely abolished the TsSP-induced LFA-1 avidity (Fig. 7c), whereas LFA-1 affinity remained unaffected (Fig. 7d). Moreover, the TsSP-induced TNF- $\alpha$ and IL-10 secretion was severely impaired in PKC $\delta$ deficient monocytes (Fig. 7e, f). Together, these findings indicate that TsSP predominantly exerts its effect on human monocytes via PKC $\delta$ signalling.

\section{Discussion}

Helminths have the capacity to survive in their host by modulating the immune response towards a tolerant or non-inflammatory response. Unravelling their mechanism of action may provide a key to combat various inflammatory diseases. Indeed, recent clinical trials in MS patients show beneficial results of administration of probiotic helminths, and their protective mechanism of action has been largely attributed to the induction of regulatory immune responses via adaptive immunity. However, as innate immune cells like monocytes belong to the first responders to parasite infections and are regarded as crucial players in MS pathogenesis, we here evaluated the effect of the porcine nematode Trichuris suis soluble products (TsSP) on monocyte phenotype and function. We demonstrate that TsSP affect classical monocytes, by inducing a shift from classical to nonclassical cells with decreased adhesion to and migration across a human in vitro blood-brain barrier (BBB). 


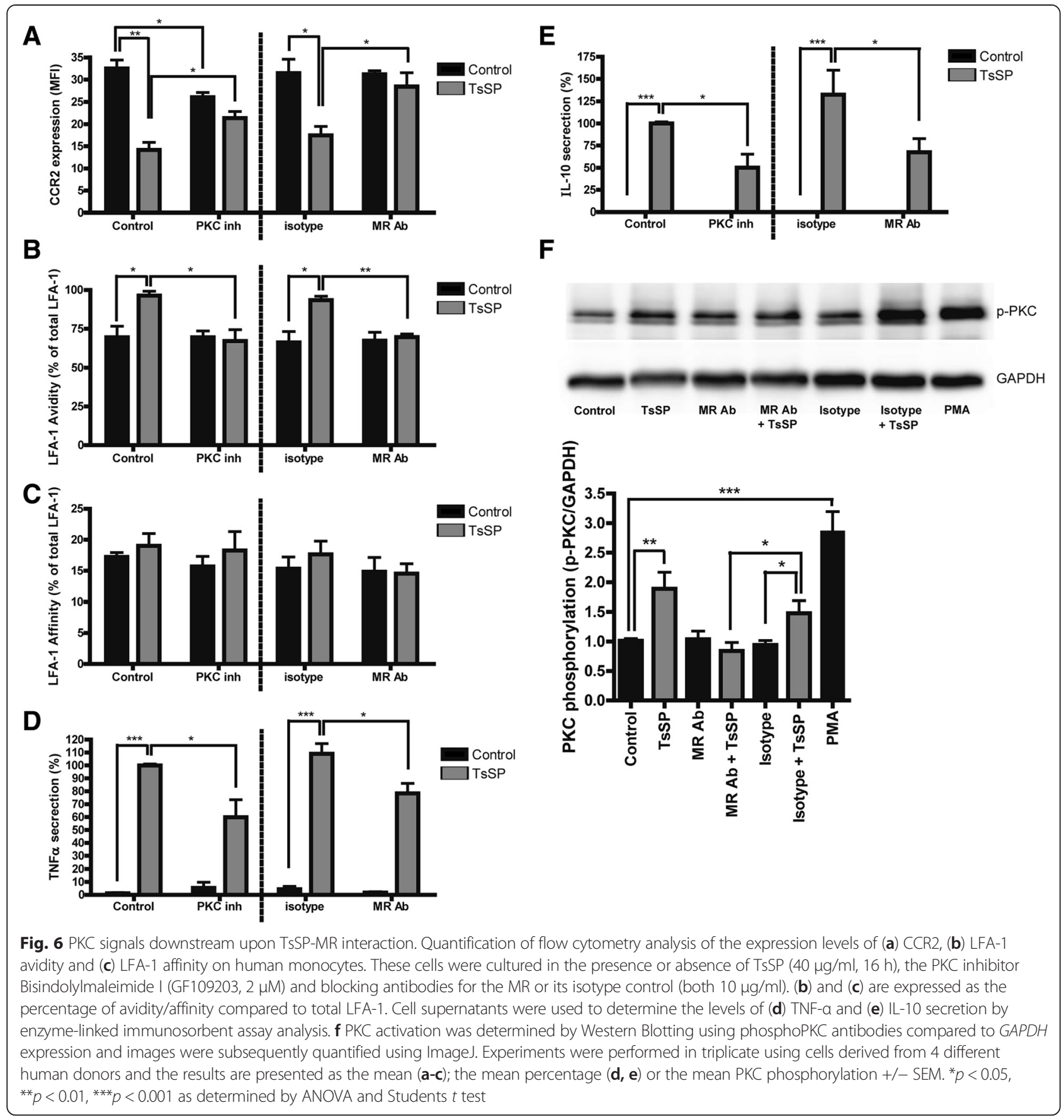

Mechanistically, we show that TsSP interacts with the mannose receptor (MR, CD206) and we identified PKC and in particular PKC $\delta$ as the main signalling molecule downstream of the MR responsible for the observed altered monocyte functions. Overall, this study provides mechanistic insights into the effect of TsSP on the innate immune response, which further unravels their protective effect to combat various inflammatory diseases, including MS.
During infection, T. suis helminths reside in the highly vascularised abdominal regions were they constantly secrete soluble products and dampen host immune responses [37]. We have previously shown that secreted products of $T$. suis can reduce the barrier integrity of intestinal epithelial cells [38], thereby facilitating the entry of helminth products into the lamina propria as well as the vasculature. Herein, circulating monocytes encounter these products and in the current study we have mimicked 


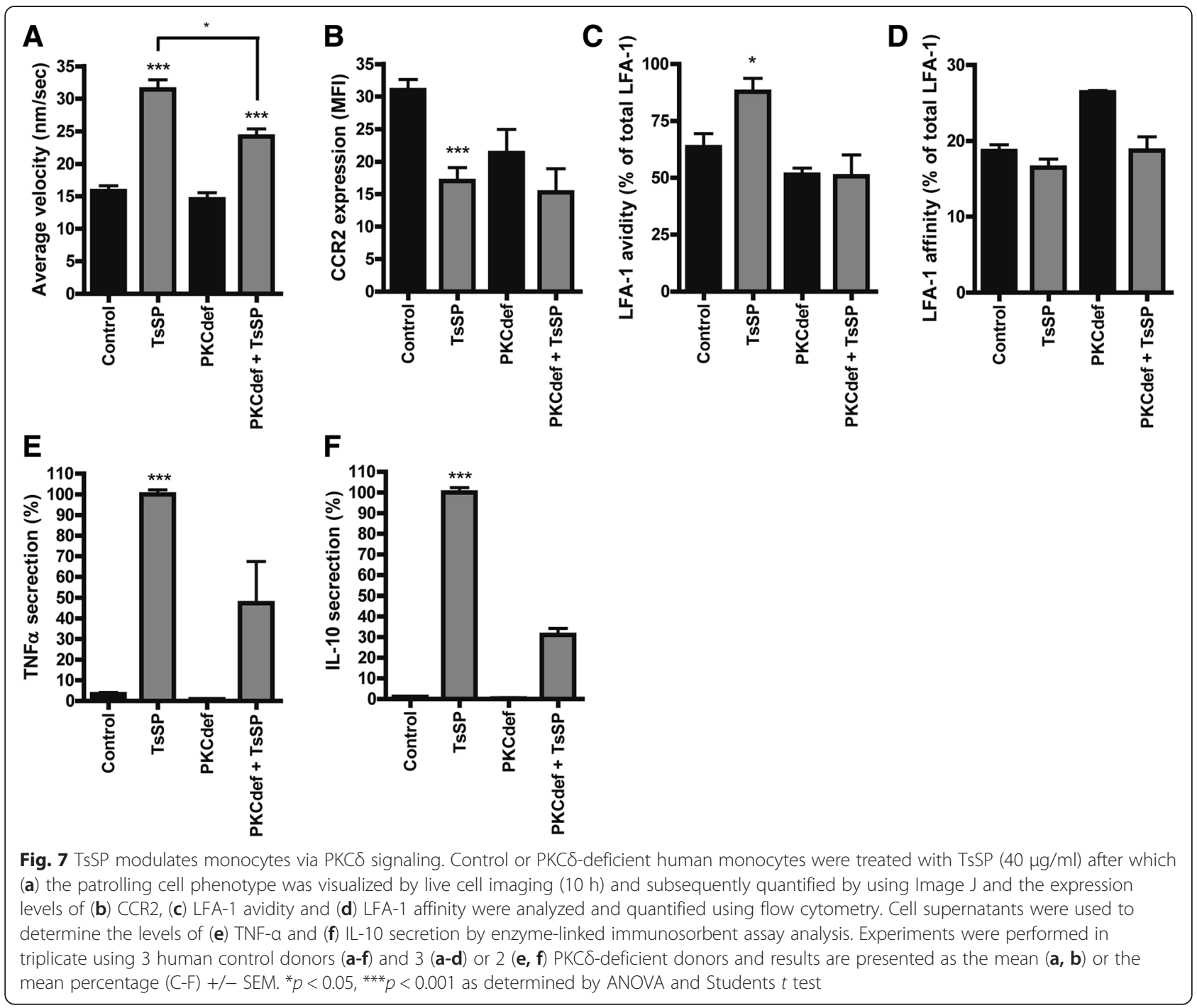

this initial encounter. We here show that TsSP treatment affects classical monocytes by reducing their CCR2 expression and inducing a shift into non-classical monocytes. At the functional level, we demonstrate that TsSP potently reduce monocyte adhesion to and transmigration across the BBB in vitro. Noteworthy, the TsSP induced effect on monocyte adhesion seems contradicting to the observed TsSP-induced LFA-1 avidity changes in human monocytes. However, it must be noted that firm adhesion requires both LFA-1 avidity and affinity conformational changes [39], and we therefore propose that the observed TsSP-induced LFA-1 avidity alone is not sufficient to enhance the adhesion of monocytes to brain endothelial cells. A key pathological hallmark of MS lesion formation is the central nervous system (CNS) entry of monocytederived macrophages across the vasculature, as these infiltrated cells are associated with axonal loss, astrogliosis and neurodegeneration [11]. We have previously shown that CCL2, the ligand for CCR2 is highly expressed by brain endothelial cells from MS patients [30] and several other studies have indicated that the CCL2/CCR2 axis is crucial for the entry of monocytes to the site of inflammation [40]. Moreover, mice that lack CCR2 are resistant to the induction of EAE $[41,42]$, thereby revealing the relevance for the CCL2/CCR2 axis for neuro-inflammation. Importantly, CCR2-deficient leukocytes show impairments in their adhesion and migration capacity across endothelial cells [43]. Therefore, the observed TsSP effects on monocyte adhesion and transmigration may be largely attributed to a loss of CCR2 expression.

Compared to control, MS patient monocytes display a pronounced inflammatory profile, including high expression of CD40, CD86, CD64 and CCR2 [19]. The identification of molecules and mechanisms that can skew these inflammatory cells into anti-inflammatory [16] and wound healing [20] patrolling cells may 
provide novel ways to combat disease progression. In the current study, we provide first evidence that TsSP may exert such a shift in monocyte phenotype. Interestingly, in vivo studies have indicated that the murine equivalent (GR $1^{\text {low }}$ monocytes [16] or type II monocytes) can significantly reduce clinical signs of EAE [44, 45], further illustrating that a shift in the monocyte population from inflammatory to anti-inflammatory cells can be regarded as a promising therapeutic avenue. In that way, TsSP may act in a similar way as a currently approved MS drug glatiramer acetate (Copaxone), which promotes the development of anti-inflammatory monocytes in vivo [45]. Next to that, it is tempting to speculate that patrolling monocytes may resemble the precursors for alternatively activated macrophages (AAM or M2 macrophages) that are required for proper tissue restoration after an inflammatory event [46]. Although this differentiation has been shown for murine Ly6C $\mathrm{C}^{-}$monocytes [47], this transition in a human setting remains to be shown.

Helminths and their soluble products are glycosylated, and the glycans of several helminth species have been shown to be essential for the induction of Th2 responses [48]. We here extend these initial findings and show that periodate-sensitive glycan moieties on TsSP are required for the observed altered monocyte functions. These glycan moieties can be recognized by specific glycan-binding proteins such as C-type lectins (CLRs), and in the current study we identified the MR as the TsSP-interacting human monocytic receptor. The MR is a type- 1 membrane protein with a single transmembrane domain and a cytoplasmic domain that mediates receptor internalisation and recycling [49]. It can bind a wide variety of exogenous and endogenous molecules and therefore is thought to mediate both homeostatic and immune processes [50]. However, as MR-deficient animals do not display enhanced susceptibility to pathogens that contain MR-ligands [51, 52], its function in host defence is not fully understood. It has been shown that Trichuris muris, which is the murine equivalent of $T$. suis, was able to interact with the MR on macrophages. However, experiments with MR-deficient animals showed that this interaction was not needed for the expulsion of the parasite [53]. The diverse secretome of helminths [54] may interact with various cellular receptors, but based on our findings we propose that the antiinflammatory effect on human monocytes is largely MRdependent. It has been show that the MR is involved in the recognition and uptake of various pathogens [50] and further research is warranted to reveal which glycanligands of TsSP interact with the MR and how these molecules are further processed within the cell. Interestingly, we observed that upon MR-TsSP interaction, monocytes display increased motility and crawling behaviour, similar to the murine patrolling $\mathrm{Gr}^{-}$monocytes with antiinflammatory capacities [16]. Although it has previously been suggested that the MR may be involved in cell motility [50], these are the first data to provide actual evidence for this in a human setting.

To date, the downstream signalling pathway of the MR remains largely elusive, and in the current manuscript we unravelled that $\mathrm{PKC}$, and in particular PKC $\delta$ signals downstream of the MR upon TsSP interaction, thereby regulating LFA-1 activation, CCR2 expression and cytokine secretion. PKC isoforms are important intracellular signalling molecules involved in cell differentiation, migration, proliferation and activation [55]. Our findings confirm previous observations that CCR2 expression and LFA-1 avidity are regulated by PKC [33, 34] but extend these findings to i) a human setting and ii) by the identification of the upstream cellular receptor (MR) as well as the identification of $\mathrm{PKC} \delta$-signaling in this regard. To date, the role of PKC $\delta$ in human monocytes has hardly been studied due the fact that specific PKC $\delta$ inhibitors are currently not available [36] as well as the general appreciation that other PKC isoforms (PKC $\alpha$ and $\mathrm{PKC} \beta$ ) are the more dominant members present in monocytes [56]. Importantly, there are only five patients worldwide known with a mutation in the PRKCD gene $[21,22]$. In the current study, we used monocytes from three different PKC $\delta$-deficient patients, which aided our studies in showing that TsSP exerts its effect on human monocytes predominantly via PKC $\delta$ signalling.

\section{Conclusion}

In conclusion, we here show a potent anti-inflammatory effect of TsSP on human monocytes and identified the MR as the TsSP-interacting receptor as well as specific downstream signalling via PKC. In turn, this study provides first insight into the beneficial effect of TsSP on the innate immune system, which together with our recent described effects of TsSP on the adaptive immune responses $[7,9]$ provides comprehensive insight in their therapeutic potential to combat various inflammatory diseases, including MS. Further research is warranted to unravel the identity of the TsSP molecules with immunomodulating capacity, in order to more specifically dampen pathogenic immune responses under inflammatory conditions and subsequently avoiding the use of whole worms as a therapy.

\section{Additional files}

Additional file 1: Video 1 and 2 are live cell imaging movies that show monocyte motility under control (video 1) or TsSP conditions (video 2).

Additional file 2: shows MR and Dectin-2 protein (2a) and gene expression (2b) levels in human monocytes upon TsSP treatment.

Additional file 3: shows the results from a monocyte motility assay (3a) and monocyte transendothelial migration assay (3b) to asses the role of Dectin- 2 in the TsSP-induced monocyte functional alterations. 


\section{Abbreviations}

7AAD: 7-Aminoactinomycin D; BBB: Blood brain barrier; CCL2: C-C chemokine ligand type 2; CCR2: C-C chemokine receptor type 2; CNS: Central nervous system; $C T$ : Chymotrypsin; CLR: C type lectin receptor; $C X_{3} C R 1$ : Fractalkine receptor; DC: Dendritic cell; EAE: Experimental autoimmune encephalomyelitis; EC: Endothelial cell; ICAM-1: Intercellular adhesion molecule 1; IL: Interleukin; LFA-1: Lymphocyte function-associated antigen 1; MR: Mannose receptor; MS: Multiple sclerosis; PI: Periodate; PKC: Protein kinase C; ROS: Reactive oxygen species; SOCS1: Suppressor of cytokine signaling 1; TGF $\beta$ : Transforming growth factor beta; TNF-a: Tumor necrosis factor alpha; TsSP: Trichuris suis soluble products; VCAM-1: Vascular cell adhesion protein 1.

\section{Competing interests}

The authors declare that they have no competing interests.

\section{Authors' contribution}

Study design: GK, IVD, CDD; Study coordination: GK, IVD, CCD; Manuscript preparation: GK, IVD, CCD; Monocyte isolation: $L C L, E F W, K B, A B, K S$, TKvdB; Flow cytometry: GK, LCL, JJK; RT-PCR, ELISA and WB: LCL, TL, AME; Live cell imaging: $G K, L C L, R B, T L, J D v B, M v E ;$ Monocyte adhesion and migration: GK, AME, SMAvdP, SJVV, HEdV; Manuscript review: RB, JJK, SJVV, IVD, CCD, KS, HEdV, JDvB, RDC. All authors read and approved the final manuscript.

\section{Acknowledgements}

This work was supported by grants from the National Multiple Sclerosis Society (PP1686) and the Dutch MS Research Foundation (MS 11-771) to IVD, and from the National Institute of Health Grant Al101982 to RDC.

\section{Author details}

${ }^{1}$ Department of Molecular Cell Biology and Immunology, Neuroscience Campus Amsterdam, VU University Medical Center, P.O. Box 70571007 MB Amsterdam, The Netherlands. ${ }^{2}$ Divison of Neonatology, Paediatric Intensive Care and Neuropaediatrics, Department of Paediatrics and Adolescent Medicine, Medical University of Vienna, Vienna, Austria. ${ }^{3}$ CeMM Research Center for Molecular Medicine of the Austrian Academy of Sciences, Vienna, Austria. ${ }^{4}$ Hôpital Femme Mère Enfant, Hospices Civils de Lyon and Université de Lyon, Lyon, France. ${ }^{5}$ Department of Blood Cell Research, University of Amsterdam, Amsterdam, The Netherlands. ${ }^{6}$ Department of Molecular Cell Biology, Sanquin Research and Landsteiner Laboratory, Academic Medical Center, University of Amsterdam, Amsterdam, The Netherlands. ${ }^{7}$ Department of Biochemistry, Emory University School of Medicine, Atlanta, USA.

\section{Received: 9 July 2015 Accepted: 10 July 2015}

Published online: 25 July 2015

\section{References}

1. Frohman EM, Racke MK, Raine CS (2006) Multiple sclerosis-the plaque and its pathogenesis. N Engl J Med 354:942-955

2. Peterson JW, Trapp BD (2005) Neuropathobiology of multiple sclerosis. Neurol Clin 23:107, vii

3. Fleming JO, Cook TD (2006) Multiple sclerosis and the hygiene hypothesis. Neurology 67:2085-2086

4. Correale J, Farez MF (2011) The impact of parasite infections on the course of multiple sclerosis. J Neuroimmunol 233:6-11

5. Rosche B, Wernecke KD, Ohlraun S, Dorr JM, Paul F (2013) Trichuris suis ova in relapsing-remitting multiple sclerosis and clinically isolated syndrome (TRIOMS): study protocol for a randomized controlled trial. Trials 14:112

6. Jex AR, Nejsum P, Schwarz EM, Hu L, Young ND, Hall RS et al (2014) Genome and transcriptome of the porcine whipworm Trichuris suis. Nat Genet 46:701-706

7. Kuijk LM, Klaver EJ, Kooij G, van der Pol SM, Heijnen P, Bruijns SC et al (2012) Soluble helminth products suppress clinical signs in murine experimental autoimmune encephalomyelitis and differentially modulate human dendritic cell activation. Mol Immunol 51:210-218

8. Banchereau J, Steinman RM (1998) Dendritic cells and the control of immunity. Nature 392:245-252

9. Klaver EJ, Kuijk LM, Laan LC, Kringel H, van Vliet SJ, Bouma G et al (2013) Trichuris suis-induced modulation of human dendritic cell function is glycan-mediated. Int J Parasitol 43:191-200
10. Ottow MK, Klaver EJ, van der Pouw Kraan TC, Heijnen PD, Laan LC, Kringel H et al (2014) The helminth Trichuris suis suppresses TLR4-induced inflammatory responses in human macrophages. Genes Immun 15:477-486

11. Bruck W (2005) The pathology of multiple sclerosis is the result of focal inflammatory demyelination with axonal damage. J Neurol 252(Suppl 5):v3-v9

12. Ginhoux F, Jung S (2014) Monocytes and macrophages: developmental pathways and tissue homeostasis. Nat Rev Immunol 14:392-404

13. Ziegler-Heitbrock L (2007) The CD14+ CD16+ blood monocytes: their role in infection and inflammation. J Leukoc Biol 81:584-592

14. Geissmann F, Jung S, Littman DR (2003) Blood monocytes consist of two principal subsets with distinct migratory properties. Immunity 19:71-82

15. Shi C, Pamer EG (2011) Monocyte recruitment during infection and inflammation. Nat Rev Immunol 11:762-774

16. Cros J, Cagnard N, Woollard K, Patey N, Zhang SY, Senechal B et al (2010) Human CD14dim monocytes patrol and sense nucleic acids and viruses via TLR7 and TLR8 receptors. Immunity 33:375-386

17. Auffray C, Fogg D, Garfa M, Elain G, Join-Lambert O, Kayal S et al (2007) Monitoring of blood vessels and tissues by a population of monocytes with patrolling behavior. Science 317:666-670

18. Greenwood J, Heasman SJ, Alvarez JI, Prat A, Lyck R, Engelhardt B (2011) Review: leucocyte-endothelial cell crosstalk at the blood-brain barrier: a prerequisite for successful immune cell entry to the brain. Neuropathol Appl Neurobiol 37:24-39

19. Chuluundorj D, Harding SA, Abernethy D, La Flamme AC (2014) Expansion and preferential activation of the CD14(+)CD16(+) monocyte subset during multiple sclerosis. Immunol Cell Biol 92:509-517

20. Nahrendorf M, Swirski FK, Aikawa E, Stangenberg L, Wurdinger T, Figueiredo JL et al (2007) The healing myocardium sequentially mobilizes two monocyte subsets with divergent and complementary functions. J Exp Med 204:3037-3047

21. Belot A, Kasher PR, Trotter EW, Foray AP, Debaud AL, Rice Gl et al (2013) Protein kinase cdelta deficiency causes mendelian systemic lupus erythematosus with B cell-defective apoptosis and hyperproliferation. Arthritis Rheum 65:2161-2171

22. Salzer E, Santos-Valente E, Klaver S, Ban SA, Emminger W, Prengemann NK et al (2013) B-cell deficiency and severe autoimmunity caused by deficiency of protein kinase $C$ delta. Blood 121:3112-3116

23. Henderson LM, Chappell JB (1993) Dihydrorhodamine 123: a fluorescent probe for superoxide generation? Eur J Biochem 217:973-980

24. García-Vallejo JJ, Van Het Hof B, Robben J, Van Wijk JA, Van Die I, Joziasse DH (2004) Approach for defining endogenous reference genes in gene expression experiments. Anal Biochem 329:293-299

25. Weksler BB, Subileau EA, Perriere N, Charneau P, Holloway K, Leveque M et al (2005) Blood-brain barrier-specific properties of a human adult brain endothelial cell line. FASEB J 19:1872-1874

26. Schreibelt G, Kooij G, Reijerkerk A, van Doorn R, Gringhuis SI, van der Pol S (2007) Reactive oxygen species alter brain endothelial tight junction dynamics via RhoA, PI3 kinase, and PKB signaling. FASEB J 21(13):3666-3676

27. Lu C, Ferzly M, Takagi J, Springer TA (2001) Epitope mapping of antibodies to the C-terminal region of the integrin beta 2 subunit reveals regions that become exposed upon receptor activation. J Immunol 166:5629-5637

28. van Kooyk $Y$, Weder $P$, Hogervorst $F$, Verhoeven AJ, van Seventer $G$, te Velde AA (1991) Activation of LFA-1 through a Ca2(+)-dependent epitope stimulates lymphocyte adhesion. J Cell Biol 112:345-354

29. Spiering D, Hodgson L (2011) Dynamics of the Rho-family small GTPases in actin regulation and motility. Cell Adh Migr 5:170-180

30. Kooij G, Kroon J, Paul D, Reijerkerk A, Geerts D, van der Pol SM et al (2014) P-glycoprotein regulates trafficking of CD8(+) T cells to the brain parenchyma. Acta Neuropathol 127:699-711

31. McGreal EP, Rosas M, Brown GD, Zamze S, Wong SY, Gordon S et al (2006) The carbohydrate-recognition domain of Dectin-2 is a C-type lectin with specificity for high mannose. Glycobiology 16:422-430

32. Chieppa M, Bianchi G, Doni A, Del PA, Sironi M, Laskarin G et al (2003) Cross-linking of the mannose receptor on monocyte-derived dendritic cells activates an anti-inflammatory immunosuppressive program. J Immunol 171:4552-4560

33. Phillips RJ, Lutz M, Premack B (2005) Differential signaling mechanisms regulate expression of $C C$ chemokine receptor-2 during monocyte maturation. J Inflamm (Lond) 2:14

34. van Kooyk Y, van Vliet SJ, Figdor CG (1999) The actin cytoskeleton regulates LFA-1 ligand binding through avidity rather than affinity changes. J Biol Chem 274:26869-26877 
35. Hardison SE, Brown GD (2012) C-type lectin receptors orchestrate antifungal immunity. Nat Immunol 13:817-822

36. Szilagyi $K$, Meijer AB, Neele AE, Verkuijlen $P$, Leitges $M$, Dabernat $S$ et al (2014) PKCdelta is dispensible for oxLDL uptake and foam cell formation by human and murine macrophages. Cardiovasc Res 104:467-476

37. Hewitson JP, Grainger JR, Maizels RM (2009) Helminth immunoregulation: the role of parasite secreted proteins in modulating host immunity. Mol Biochem Parasitol 167:1-11

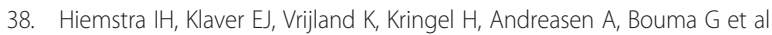
(2014) Excreted/secreted Trichuris suis products reduce barrier function and suppress inflammatory cytokine production of intestinal epithelial cells. Mol Immunol 60:1-7

39. Kinashi T (2005) Intracellular signalling controlling integrin activation in lymphocytes. Nat Rev Immunol 5:546-559

40. Matsushima K, Larsen CG, DuBois GC, Oppenheim JJ (1989) Purification and characterization of a novel monocyte chemotactic and activating factor produced by a human myelomonocytic cell line. J Exp Med 169:1485-1490

41. Fife BT, Huffnagle GB, Kuziel WA, Karpus WJ (2000) CC chemokine receptor 2 is critical for induction of experimental autoimmune encephalomyelitis. J Exp Med 192:899-905

42. Izikson L, Klein RS, Charo IF, Weiner HL, Luster AD (2000) Resistance to experimental autoimmune encephalomyelitis in mice lacking the CC chemokine receptor (CCR)2. J Exp Med 192:1075-1080

43. Kuziel WA, Morgan SJ, Dawson TC, Griffin S, Smithies O, Ley K et al (1997) Severe reduction in leukocyte adhesion and monocyte extravasation in mice deficient in CC chemokine receptor 2. Proc Natl Acad Sci U S A 94:12053-12058

44. Mikita J, Dubourdieu-Cassagno N, Deloire MS, Vekris A, Biran M, Raffard G et al (2011) Altered M1/M2 activation patterns of monocytes in severe relapsing experimental rat model of multiple sclerosis. Amelioration of clinical status by M2 activated monocyte administration. Mult Scler 17:2-15

45. Weber MS, Prod'homme T, Youssef S, Dunn SE, Rundle CD, Lee L et al (2007) Type II monocytes modulate T cell-mediated central nervous system autoimmune disease. Nat Med 13:935-943

46. Schwartz M, Baruch K (2014) The resolution of neuroinflammation in neurodegeneration: leukocyte recruitment via the choroid plexus. EMBO J $33: 7-22$

47. Italiani P, Boraschi D (2014) From Monocytes to M1/M2 Macrophages: Phenotypical vs. Functional Differentiation Front Immunol 5:514

48. Van Die I, Cummings RD (2010) Glycan gimmickry by parasitic helminths: a strategy for modulating the host immune response? Glycobiology 20:2-12

49. Martinez-Pomares L (2012) The mannose receptor. J Leukoc Biol 92:1177-1186

50. Gazi U, Martinez-Pomares L (2009) Influence of the mannose receptor in host immune responses. Immunobiology 214:554-561

51. Lee SJ, Zheng NY, Clavijo M, Nussenzweig MC (2003) Normal host defense during systemic candidiasis in mannose receptor-deficient mice. Infect Immun 71:437-445

52. Swain SD, Lee SJ, Nussenzweig MC, Harmsen AG (2003) Absence of the macrophage mannose receptor in mice does not increase susceptibility to Pneumocystis carinii infection in vivo. Infect Immun 71:6213-6221

53. deSchoolmeester ML, Martinez-Pomares L, Gordon S, Else KJ (2009) The mannose receptor binds Trichuris muris excretory/secretory proteins but is not essential for protective immunity. Immunology 126:246-255

54. Ditgen D, Anandarajah EM, Meissner KA, Brattig N, Wrenger C, Liebau E (2014) Harnessing the helminth secretome for therapeutic immunomodulators. Biomed Res Int 2014:964350

55. Bertram A, Ley K (2011) Protein kinase C isoforms in neutrophil adhesion and activation. Arch Immunol Ther Exp (Warsz) 59:79-87

56. Chang ZL, Beezhold DH (1993) Protein kinase $C$ activation in human monocytes: regulation of PKC isoforms. Immunology 80:360-366

\section{Submit your next manuscript to BioMed Central and take full advantage of:}

- Convenient online submission

- Thorough peer review

- No space constraints or color figure charges

- Immediate publication on acceptance

- Inclusion in PubMed, CAS, Scopus and Google Scholar

- Research which is freely available for redistribution

Submit your manuscript at www.biomedcentral.com/submit 